\title{
Developing ecospheres on transiently habitable planets: the genesis project
}

\author{
Claudius Gros ${ }^{1}$ (iD
}

Received: 8 June 2016 / Accepted: 19 August 2016 / Published online: 5 September 2016

(c) The Author(s) 2016. This article is published with open access at Springerlink.com

\begin{abstract}
It is often presumed, that life evolves relatively fast on planets with clement conditions, at least in its basic forms, and that extended periods of habitability are subsequently needed for the evolution of higher life forms. Many planets are however expected to be only transiently habitable. On a large set of otherwise suitable planets life will therefore just not have the time to develop on its own to a complexity level as it did arise on earth with the cambrian explosion. The equivalent of a cambrian explosion may however have the chance to unfold on transiently habitable planets if it would be possible to fast forward evolution by 3-4 billion years (with respect to terrestrial timescales). We argue here, that this is indeed possible when seeding the candidate planet with the microbial lifeforms, bacteria and unicellular eukaryotes alike, characterizing earth before the cambrian explosion. An interstellar mission of this kind, denoted the 'Genesis project', could be carried out by a relatively low-cost robotic microcraft equipped with a on-board gene laboratory for the in situ synthesis of the microbes.

We review here our current understanding of the processes determining the timescales shaping the geo-evolution of an earth-like planet, the prospect of finding Genesis candidate planets and selected issues regarding the mission layout. Discussing the ethical aspects connected with a Genesis mission, which would be expressively not for human benefit, we will also touch the risk that a biosphere incompatibility may arise in the wake of an eventual manned exploration of a second earth.
\end{abstract}

Keywords Astrobiology $\cdot$ Habitable planets $\cdot$ Interstellar missions

C. Gros

gros07@itp.uni-frankfurt.de

1 Institute for Theoretical Physics, Goethe University Frankfurt, Germany

\section{Introduction}

Three ongoing lines of research have progressed in the last years to a point which allows us to assess now the feasibility of sending out interstellar probes with the mission of bringing life to otherwise barren exoplanets.

In first place comes here the insight from exoplanet search efforts, that the diversity of the hitherto discovered exoplanetary systems is very high. This implies, in particular, that no two habitable planets may be alike (Güdel et al. 2014) and that there will be many planets having only limited periods of habitability. There may hence exist in our galaxy a plethora of planets where life could truly thrive, having however not enough time to fully develop on its own. The key idea of the Genesis project is to bring life to these kind of exoplanets.

The Genesis project is based furthermore on the evolving consensus (Long 2011; Gilster 2015; Worden et al. 2016), that robotic interstellar missions may be realizable within a foreseeable future. A conceivable scenario would be in this context to accelerate lightweight interstellar probes with ground- or orbit-based arrays of powerful lasers (Zhang et al. 2015; Brashears et al. 2015). Decelerating could then be achieved, on arrival, using magnetic and/or electric sails (Zubrin and Andrews 1991; Perakis and Hein 2016).

The progress (Gibson et al. 2010; Hutchison et al. 2016) achieved recently in creating synthesized and minimal (in terms of the genome) cells, indicates furthermore that humanity will acquire most probably already within a few decades the capability to synthesize a vast palette of life forms from scratch. We can hence envision that a Genesis probe would be able to cultivate in situ many different types of microbes using a robotic gene laboratory. 
The Genesis project consists hence of three steps.

- Searching for transiently habitable planets.

- Sending interstellar robotic crafts for detailed investigations.

- Seeding the candidate planet with in situ synthesized lifeforms.

In this study we will review the prospects of discovering Genesis candidate planets, the time scale for evolutionary speedup one may realistically hope to achieve and the time scales the Genesis process may take to unfold. The Genesis project comes of course with serious ethical caveats regarding in particular planetary protection, which we will also discuss.

The Genesis project is in first place not for human benefit. The key idea is to initiate a self-sustained evolutionary process, which will then carry the developing biosphere of the host planet to its own future. Later stage human interventions are not excluded, but not necessary. The same holds for an eventual human settlement of the candidate planet. In this context we will also discuss the prospective that biosphere incompatibilities may accompany manned missions to second-earth like exoplanets.

\subsection{Genesis candidate planets}

We currently do not know whether the bio-geological evolution of earth has been fast or slow in relation to evolutionary processes on other habitable planets. We know however, that the timescale of major geo-evolutionary steps has been typically of the order of one or more billion years $(\mathrm{Ga})$. Taking hence one $\mathrm{Ga}$ as a reference time we may consider a planet to be transiently habitable if the time span it remains in the habitable zone (HZ) ranges from at least a few hundred million years to about $1-2 \mathrm{Ga}$.

There are various conceivable scenarios why a planet may be habitable for a finite, but prolonged period.

- Shift of the habitable zone.

Main sequence stars as our sun, which was initially about $30 \%$ less luminous, become brighter with the eons passing. A planet starting out at inner edge of the $\mathrm{HZ}$ may hence become eventually too hot (Rushby et al. 2013). An initially too far away planet may reversely warm up, but possibly only after a few Ga have passed. In the later case not enough time for the unfolding of a full-fledged evolutionary process may be left.

- Long-term orbital instabilities.

Several kinds of long-term orbital instabilities, like the Hill and the Lagrange instability discussed in Sect. 3.2 and 3.3, may throw a planet out of the habitable zone or, reversely, promote a planet into the HZ (Jones et al. 2006). Habitability may also be interrupted by later stage orbital resonances, as the one which has possibly caused the late heavy bombardment of our home planet (compare Sect. 2.2).

- Indigenous processes.

An initially habitable planet may become inhabitable also all by itself. Various indigenous processes are conceivable in this context.

- Plate tectonic may cease functioning after a certain time, giving way to the type of stagnant lid magma convection presumable in place on Venus. Episodic overturns of the crust may then result in global resurfacing events.

- Shifts in the $\mathrm{CO}_{2}$ balance could lead to the complete depletion of atmospheric $\mathrm{CO}_{2}$ levels. On earth this is actually happening, as discussed in Sect. 2.6, albeit only very slowly. A continuous accumulation of $\mathrm{CO}_{2}$ could result on the other side in either a catastrophic runaway, or in a welcome Greenhouse effect.

Reviewing the prospects of discovering Genesis candidate we will rely in part on an analysis of the timeline of the geo-evolutionary history of our home planet. Of particular interest for the Genesis project are here the involved time scales and the question, whether alternative evolutionary routes would have been possible.

\section{Terrestrial timescales}

A central question of the present study regards the time one may expect a Genesis process will need to unfold on an exoplanet. As a backdrop to this question we start with a short review of some of the key events in the geo-biological evolution of earth, where a self-organized Genesis process is known to have occurred. Times will be given either in Gigayears $\left(10^{9} \mathrm{Ga}\right)$ or Mega-years $\left(10^{6} \mathrm{Ma}\right)$. Some of the key events shaping our home planet are shown on scale in Fig. 1.

Shortly after earth was formed together with most of the solar system about $4.6 \mathrm{Ga}$ ago, an impact with a Mars-size object led to the creation of the moon (Canup and Righter 2000). Our moon is exceptionally large and it is established that its size helps to stabilize the rotation axis (the obliquity) of earth (Laskar et al. 1993). Seasonal variability (between sumer and winter) would be otherwise substantially larger. The presence of the moon is however not a precondition for habitability per se.

\subsection{5-4.0 Ga: the hadean $\mathrm{CO}_{2}$ sequestration}

It is not known how wet earth initially was, viz which percentage of today's water was initially present and to which extent water was brought to young earth from further-out solar objects (Drake and Righter 2002). It is however believed that the outgasing of the volatiles from the initially hot magma lead to a dense $\mathrm{CO}_{2}$ atmosphere (Sleep 2010) (about 
Fig. 1 Some major milestones in the history of our home planet (time in $10^{9}$-years, Ga).

A one-shot Genesis probe may achieve an evolutionary fast forward for the candidate planet to eukaryotic life, viz by about three Ga. The blue bars denote episodes of global glaciation (snowball earth). Huronian, Sturtian and Marinoan at $2.27 \mathrm{Ga}$ and $716.5 / 635 \mathrm{Ma}$ (Tang and Chen 2013; Macdonald et al. 2010)

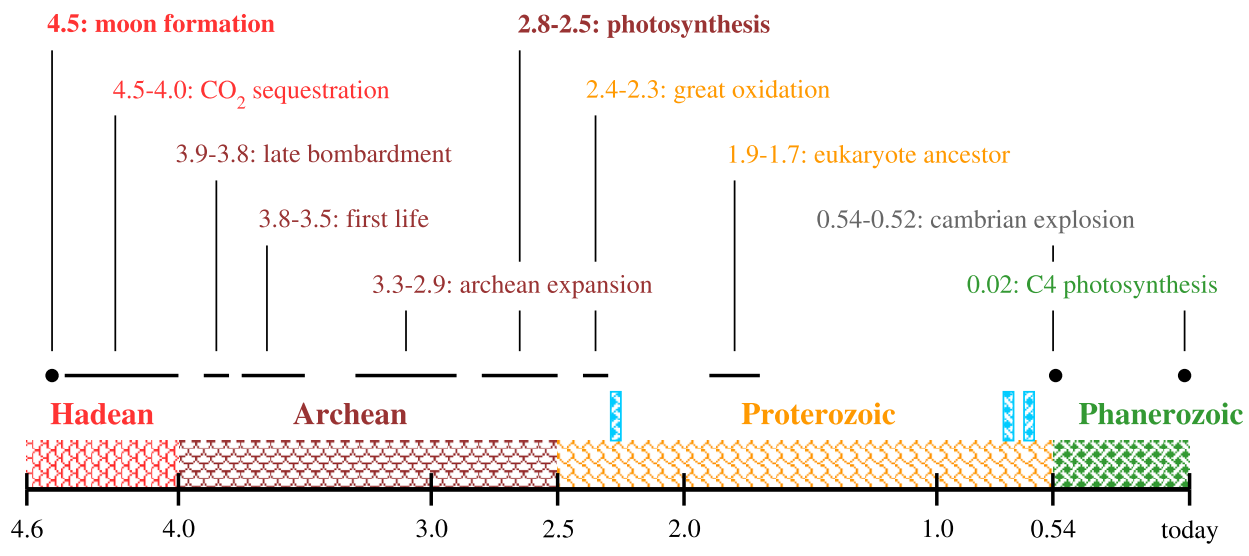

100 bar of $\mathrm{CO}_{2}$, as for today's Venus), which, in turn, prevented earth to cool below $500{ }^{\circ} \mathrm{C}$ after the formation of the moon. A liquid ocean of some extent would however been present despite the elevated surface temperature as a consequence of the likewise increased atmospheric pressure.

Any planet hoping for an earth-like habitability needs to rid itself of its primordial $\mathrm{CO}_{2}$ atmosphere. This was achieved on earth by the carbonation

$\mathrm{CaSiO}_{3}+\mathrm{CO}_{2} \rightarrow \mathrm{CaCO}_{3}+\mathrm{SiO}_{2}$

of silicates and the subsequent subduction of carbonized rocks (the Urey weathering reaction). It is unsure how long it actually took, possibly up to the end of the Hadean (4 Ga), for the subducted crust to sequester $\mathrm{CO}_{2}$ to levels of only 10-100 times today's levels (about 0.0004 bar).

The hadean $\mathrm{CO}_{2}$ sequestration was a massive process. For a perspective we note that the present day rate of $\mathrm{CO}_{2}$ sequestration by modern plate tectonics is of the order of $3.3 \times 10^{18} \mathrm{~mol} / \mathrm{Ma}$ (Sleep and Zahnle 2001). With $100 \mathrm{bar}$ $\mathrm{CO}_{2}$ corresponding to $12000 \times 10^{18} \mathrm{~mol}$ this implies, that the sequestration of the primordial $\mathrm{CO}_{2}$ at present-day rates would have taken (12000/3.3) Ma, viz 3.6 Ga. Substantially faster subduction processes must have been consequently at work in the early Hadean (Sleep et al. 2014).

It is reasonable to expect $\mathrm{CO}_{2}$ sequestration to be generically vigorous on potentially habitable rocky planets.

- The essentially unlimited reservoir of silicates rocky planets like the earth dispose of, compare Table 1, allows to sequester basically all $\mathrm{CO}_{2}$ from the atmosphere. The steady-state level of atmospheric $\mathrm{CO}_{2}$ will then result on habitable planets from the balance between volcanic outgasing and ongoing sequestration (the inorganic carbon cycle) (Sleep and Zahnle 2001).

- The hadean $\mathrm{CO}_{2}$ sequestration occurred at a time when earth dissipated its internal heat by bottom-up mantle convection, viz when modern plate tectonics was most probably not yet at work (Korenaga 2013). We will come back to this issue in Sect. 3.
Table 1 The mol-abundances (relative number of atoms, not weight; in percentage) of some selected elements. For the solar system (disregarding Hydrogen $\left[{ }^{*}\right]$ and Helium $\left[{ }^{\sharp}\right]$ ) (Lodders et al. 2009), the earth (all) (McDonough and Sun 1995), the crust (of the earth) (Lide and Fredrikse 2008), and for the human body (Lide and Fredrikse 2008). Note that the crust is weakly reducing in the sense that the available oxygen is nearly enough to oxidize all other elements via $\mathrm{Si}+\mathrm{O}_{2} \rightarrow \mathrm{SiO}_{2}, 4 \mathrm{Al}+3 \mathrm{O}_{2} \rightarrow 2 \mathrm{Al}_{2} \mathrm{O}_{3}$, etc. $92.1 \%$ and $7.8 \%$ of the overall number of atoms of the solar system are Hydrogen and Helium atoms respectively

\begin{tabular}{cllcccc}
\hline $\mathrm{Z}$ & & Element & Solar & Earth & Crust & Body \\
\hline 1 & $\mathrm{H}$ & Hydrogen & {$\left[^{*}\right]$} & 0.67 & 2.9 & 62.5 \\
2 & $\mathrm{He}$ & Helium & {$\left[^{\sharp}\right]$} & & & \\
6 & $\mathrm{C}$ & Carbon & 25.05 & 0.16 & 0.035 & 11.6 \\
7 & $\mathrm{~N}$ & Nitrogen & 7.39 & 0.005 & 0.003 & 1.2 \\
8 & $\mathrm{O}$ & Oxygen & 54.70 & 48.3 & 59.9 & 24.1 \\
12 & $\mathrm{Mg}$ & Magnesium & 3.59 & 16.5 & 2.0 & 0.007 \\
13 & $\mathrm{Al}$ & Aluminum & 0.29 & 1.5 & 6.3 & \\
14 & $\mathrm{Si}$ & Silicon & 3.48 & 15.0 & 20.9 & 0.006 \\
15 & $\mathrm{P}$ & Phosphorus & 0.03 & 0.1 & 0.07 & 0.22 \\
16 & $\mathrm{~S}$ & Sulfur & 1.47 & 0.52 & 0.023 & 0.04 \\
19 & $\mathrm{~K}$ & Potassium & 0.01 & 0.01 & 1.1 & 0.03 \\
20 & $\mathrm{Ca}$ & Calcium & 0.21 & 1.1 & 2.2 & 0.22 \\
26 & $\mathrm{Fe}$ & Iron & 2.95 & 14.9 & 2.1 & 0.0007 \\
\hline
\end{tabular}

It is not clear whether Venus had ever been able to start the process of $\mathrm{CO}_{2}$ sequestration even when starting out with earth-like conditions. It may have been, that its initial magma ocean took substantially longer time (100 Ma instead of 1-4 Ma, as for earth) to solidify (Hamano et al. 2013) and that Venus may have had lost most of its primordial water by that time through hydrodynamic escape (Hamano et al. 2013; Leconte et al. 2013; Kasting et al. 2015). The mechanism involves water to rise to the stratosphere and to photodissociate via $\mathrm{H}_{2} \mathrm{O}+$ light $\rightarrow \mathrm{H}_{2}+\mathrm{O}$. The free hydrogen molecules then escape the pull of gravity, being too light for a rocky planet, dragging some of the oxygen with it. This process led on Venus to the loss of an ocean 
worth of water, shutting down also the carbonation of silicate rocks via the Urey weather reaction (1), which needs in turn the formation of carbonic acid $\mathrm{CO}_{2}+\mathrm{H}_{2} \mathrm{O} \rightarrow \mathrm{H}_{2} \mathrm{CO}_{3}$ and hence the presence of liquid water in an intermediate step. The concentration of hydrogen bearing molecules like $\mathrm{H}_{2} \mathrm{O}$ and $\mathrm{NH}_{4}$ is on the other side very low in the stratosphere of earth, at least nowadays, and such the loss of $\mathrm{H}_{2}$ (Catling et al. 2001).

\subsection{9-3.8 Ga: the late heavy bombardment}

After the formation of the moon not much happened apart from the ongoing $\mathrm{CO}_{2}$ sequestration for about $600 \mathrm{Ma}$. Then, by 3.9-3.8 Ga, the late heavy bombardment (LHB) took place in the form of a cataclysmic wave of planetesimals (both asteroids and comets) battering earth together with the entire inner solar system (Gomes et al. 2005). An event like the LTB would eradicate all higher life forms on a planet, if existing, but it would not sterilize the planet altogether. The LTB may have been delivering in addition a certain fraction of the water present nowadays on earth $\left(78 \times 10^{21} \mathrm{~mol} \mathrm{H}_{2} \mathrm{O}\right.$ in the ocean alone), without affecting otherwise the habitability of the planet.

The late heavy bombardment did originate, as far as we know, from an instability of a disc of planetesimals left over from the formation of the solar system. What is interesting is, that such disks must have continued to exist long after the formation of the solar system and that the instability occurred with a huge delay. A possible scenario for this to happen is illustrated in Fig. 2 (the Nice model Gomes et al. 2005; Tsiganis et al. 2005; Bottke et al. 2012). It assumes that a 2:1 orbital resonance (in terms of their respective orbital periods) did built up due to the migration of Jupiter and Saturn, which were in turn caused by the interaction with the then still existing outer discs of planetesimals. The orbits of both Jupiter and Saturn were strongly deformed at resonance, with the consequence that the disc of planetesimal was perturbed together with the then substantially denser disc of asteroids between Mars and Jupiter. Objects were subsequently thrown out of their original orbits and sent into the inner solar systems.

\subsection{3-2.9 Ga: the archean genetic expansion}

Life emerged on earth in a multi-step process which may have started quite soon after the late heavy bombardment (Bada 2004), possibly also before, having come to a completion by around $3.5 \mathrm{Ga}$ (Nisbet and Sleep 2001). Using phylogenomic methods to analyze the evolutionary history of 3983 gene families it was found (David and Alm 2011) that the de novo creation of bacterial genes was a concentrated process, the archean genetic expansion, starting around $3.3 \mathrm{Ga}$ and ending by $2.9 \mathrm{Ga}$ with the essential completion of the bacterial molecular machinery. Gene

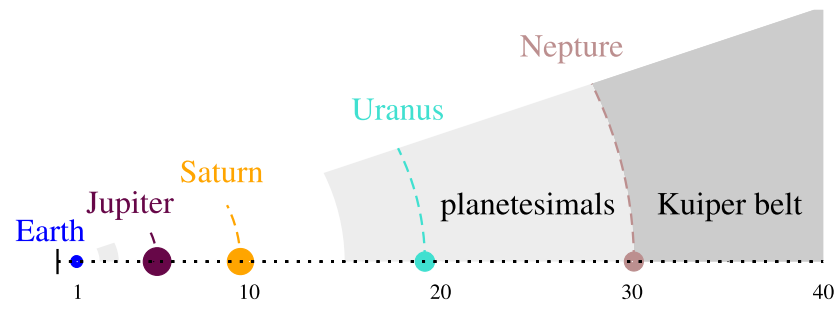

Fig. 2 An illustration for a possible scenario for the origins of the great late bombardment. Shown are today's planet positions (on scale) together with the location of the modern Kuiper belt of comets and a putative initial belt of unused planetesimals (a then still present leftover of the solar system formation). The mutual interaction with these disks may have caused Saturn and Jupiter to migrate until they entered a 2:1 orbital resonance. Today's orbital periods of 29.5 and 11.9 years, respectively for Saturn and Jupiter, are actually closer to a 3:1 resonance. An eccentricity instability would have occurred, roughly $700 \mathrm{Ma}$ after the formation of the solar system, leading to a disturbance of both the disk of planetesimal and of the asteroid belt between Mars and Jupiter (Gomes et al. 2005). The distances are given in astronomical units (earth's distance from the sun, AU)

transfer and loss tended to dominate bacterial evolution ever since (David and Alm 2011) and it is not understood why it took evolution another Ga to develop eukaryotic cells (see Sect. 2.5).

\subsection{4-2.3 Ga: the great oxidation event}

Living organisms need an energy source to power reaction pathways utilizing $\mathrm{CO}_{2}$ for the synthesis of organic molecules (carbon fixation). Early in the history of life this energy was provided mostly by chemotrophic reaction pathways (Wächtershäuser 2006), such as

$3 \mathrm{FeS}+4 \mathrm{H}_{2} \mathrm{~S}+\mathrm{CO}_{2} \rightarrow 3 \mathrm{FeS}_{2}+\mathrm{CH}_{3} \mathrm{SH}+2 \mathrm{H}_{2} \mathrm{O}$,

which uses in this case the energy released by the reaction of ferrous sulfide $(\mathrm{FeS})$ with hydrogen sulfide $\left(\mathrm{H}_{2} \mathrm{~S}\right)$. The reaction products are here pyrite $\left(\mathrm{FeS}_{2}\right)$, methanethiol $\left(\mathrm{CH}_{3} \mathrm{SH}\right)$ and water $\left(\mathrm{H}_{2} \mathrm{O}\right)$.

The global bioproductivity of chemotrophy is limited by the rate by which volcanism replenishes the primary reagents. For present day hydrothermal activity levels this implies that about $(2-20) \times 10^{12} \mathrm{~mol}$ organic $\mathrm{C}$ per year could be fixated via chemical pathways (Des Marais 2000). Today's biosphere produces by contrast around $8700 \times$ $10^{12}$ mol organic $\mathrm{C}$ per year using the light of the sun to power the photosynthesis reaction

$6 \mathrm{CO}_{2}+6 \mathrm{H}_{2} \mathrm{O}+$ light $\rightarrow \mathrm{C}_{6} \mathrm{H}_{12} \mathrm{O}_{6}+6 \mathrm{O}_{2}$.

The invention of photosynthesis occurring by the end of the archean genetic expansion, around 2.8-2.5 Ga (Des Marais 2000), possibly also later (Rasmussen et al. 2008), did therefore allow the terrestrial biosphere to expand dramatically. The balance equation (3) is in addition oxygenic in the 
sense that free $\mathrm{O}_{2}$ is produced as a waste besides glucose $\left(\mathrm{C}_{6} \mathrm{H}_{12} \mathrm{O}_{6}\right)$.

Earth changed in many ways once life started to produce oxygen in relevant quantities (Catling et al. 2001).

- The $\mathrm{Fe}^{+2}$ ions present till then in the ocean were rapidly precipitated as banded iron formations (Lyons et al. 2014). Essentially all iron-based biological pathways, like anoxic photosynthesis (Canfield 2005) (which does not produce oxygen), came hence to an abrupt end, at least on a global scale, surviving only in suitable niches.

- Non- $\mathrm{CO}_{2}$ greenhouse gases like methane $\left(\mathrm{CH}_{4}\right)$ were equally washed out from the atmosphere. The sky became clear and earth the blue planet of today. Global temperatures dropped consequently.

Taking a closer look at the elements making up our home planet, as listed in Table 1, one notices that the crust is weakly reducing in the sense that the oxidation of the other elements,

$$
\mathrm{Si}+\mathrm{O}_{2} \rightarrow \mathrm{SiO}_{2}, \quad 4 \mathrm{Al}+3 \mathrm{O}_{2} \rightarrow 2 \mathrm{Al}_{2} \mathrm{O}_{3}, \quad \text { etc. }
$$

would need somewhat more than the actually available amount of oxygen (earth as a whole would be on the other hand strongly reducing). For oxygen to accumulate in the atmosphere two things must consequently happen.

- Part of the carbon fixated via oxygenic photosynthesis must be removed from the surface via sedimentation in a process denoted the organic carbon cycle (see Fig. 3).

- All reducing elements (like iron) present on the surface must first be oxidized.

It is notoriously difficult to determine how, when and why oxygen did eventually accumulate in the atmosphere (Catling and Claire 2005; Lyons et al. 2014). It is however clear, that oxygen appeared at appreciable levels (of the order of a few percent of today's levels) in the atmosphere during the great oxidation event around 2.4-2.3 Ga. Oxygen levels didn't though remain stable afterwards, possibly plunging again for a billion years or more, with modern levels of $\mathrm{O}_{2}$ being reached only at the end of Proterozoic, viz shortly before the cambrian explosion. This second rise of $\mathrm{O}_{2}$ levels was the prerequisite for the subsequent development of animals and hence all important from a human perspective. Its ultimate causes are however not yet understood (Och and Shields-Zhou 2012).

\subsection{0-520 Ma: the cambrian explosion}

Though it is difficult, it is not impossible for bacteria to develop into at least primitive multicellular organisms (Shapiro et al. 1997). All higher plants and animals are built however out of eukaryotic cells, which dispose of a much higher complexity of internal organization. Eukaryotes evolved out of prokaryotes (bacteria and archaea) in a

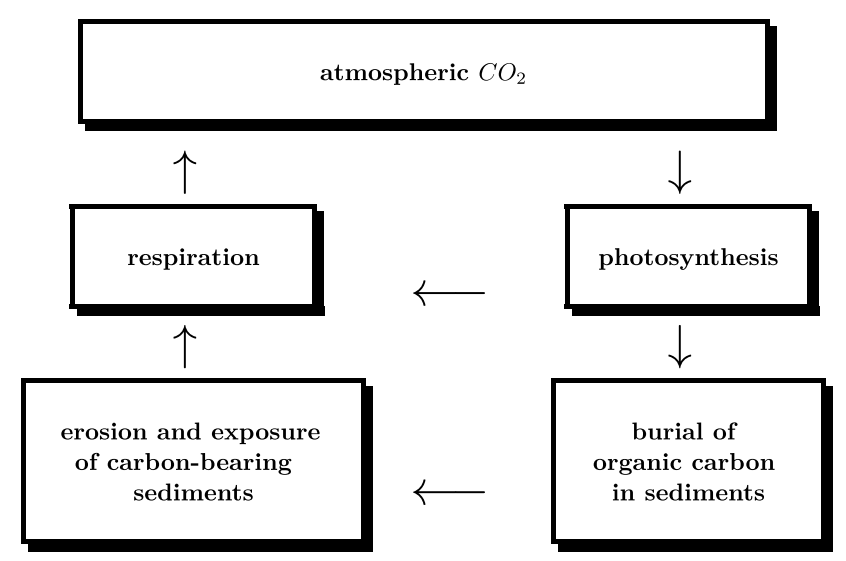

Fig. 3 Sketch of the organic carbon cycle (Jacob 1999; Sleep and Zahnle 2001). The overall amount of sedimentated organic carbon has been estimated to be of the order of $1250 \times 10^{18}$ mol. This implies, with about $37 \times 10^{18} \mathrm{~mol} \mathrm{O}_{2}$ present nowadays in the air, that earth's biosphere has produced at least $1250 / 37 \approx 34$ times more oxygen over the last four billion years than the one remaining in today's atmosphere. The present-day fluxes are: $8.7 \times 10^{15}$ mol biological $\mathrm{C}$ per year global NPP (net primary production) (Field et al. 1998), with roughly equal contributions from land and oceans, and $11 \times 10^{18} \mathrm{~mol}$ biological C/Ma buried in sediments (Sleep and Zahnle 2001)

multi-stage process ending, as determined e.g. by a multigene molecular clock analysis (Parfrey et al. 2011), by around $1.9-1.7 \mathrm{Ga}$. At that point, the last common ancestor of all eukaryotes drifted through the waters of the Proterozoic (Knoll et al. 2006).

Very little is known when the next important step in the evolution of life, sexual reproduction, did take place, (Goodenough and Heitman 2014). It could even be the case that no additional step was needed, viz that sexual reproduction is inherent to eukaryotic life per se (Speijer et al. 2015) and that the last common eukaryotic ancestor was already sexual. The advantages of sexual reproduction for multicellular organisms are in any case undisputed.

The pace of evolution did not pick up directly with the invention of eukaryotic cells. Only in the second part of the following about $1.3 \mathrm{Ga}$, the 'boring billion' (Roberts 2013), animal phyla started to diverge measurably (Blair and Hedges 2005). It is unknown (Zhang et al. 2014) which of the geo-biological events accompanying the demise of this prolonged period of stasis where the actual drivers both for ending the boring billion and for initiating the following unprecedented divergence of life known as the 'cambrian explosion' (Morris 2000; Marshall 2006).

Multicellular organisms developed not in a singular event but at least on 25 distinct occasions (Grosberg and Strathmann 2007), with the first major multicellular biota emerging being the ediacaran fauna (590-540 Ma). Of all animals crawling on the earth however only the Ecdysozoa (like centipedes) have ediacaran ancestors (Rota-Stabelli et al. 2013). All other animal phyla appeared in contrast during the following cambrian explosion, with the initial burst 
(540-520 Ma) lasting only $20 \mathrm{Ma}$. Soon after land was colonized by the ancestors of our modern plants and animals (510-470 Ma) (Rota-Stabelli et al. 2013).

\subsection{Ma: C4 photosynthesis}

Earth started, as discussed in Sect. 2.1, with something like 100 bar of $\mathrm{CO}_{2}$, which were then rapidly sequestrated. Volcanic outgasing and limited sedimentation rates kept atmospheric $\mathrm{CO}_{2}$ concentrations afterwards at levels which where still relative high in comparison to today's value (Kaufman and Xiao 2003). By $2.2 \mathrm{Ga}$ atmospheric $\mathrm{CO}_{2}$ was, for a reference, about $23 \times$ PAL (present day preindustrial atmospheric levels: $280 \mathrm{ppm}$ ) (Sheldon 2006). That changed however when global bioproductivity increased after life colonized land in the aftermath of the cambrian explosion (Igamberdiev and Lea 2006), both because of the additional carbon fixation by the land plants and because the roots of the plants intensified, in addition, the weathering of rocks and hence the amount of biologically available mineral nutrients (like phosphorus). The resulting decline of atmospheric $\mathrm{CO}_{2}$ led eventually to such low levels of $\mathrm{CO}_{2}$ (about present-day PAL) (Sage et al. 2012), that life had to readjust.

The reason is that $\mathrm{C} 3$ photosynthesis, the dominant pathway for oxygenic photosynthesis for plants, becomes at first linearly less effective with declining $\mathrm{CO}_{2}$ concentration (Collatz 1977), stopping in the end altogether once the $\mathrm{CO}_{2}$ level falls below a certain threshold (which depends in turn on other parameters like humidity, oxygen level and the like). The recent, human-induced raise of atmospheric $\mathrm{CO}_{2}$ has led conversely to an ongoing greening of earth (Sheldon 2006). By $20 \mathrm{Ma}$, with precursors starting around $30 \mathrm{Ma}$, $\mathrm{C} 4$ photosynthesis was developed as a new pathway for photosynthesis by at least 66 different terrestrial plants (Sage et al. 2012). The efficiency of $\mathrm{C} 4$ photosynthesis does not depend on $\mathrm{CO}_{2}$ partial pressures, in contrast to $\mathrm{C} 3$ photosynthesis, and it is therefore believed that its evolution constitutes a response of the biosphere to a chronic shortage of atmospheric carbon dioxide (Sage et al. 2012).

Today about $23 \%$ of the terrestrial NPP (net primary production of organic carbon) is due to $\mathrm{C} 4$ plants. During the last $0.4 \mathrm{Ma}$, when the $\mathrm{CO}_{2}$ level oscillated between $180 \mathrm{ppm}$ (during periods of extended glaciation) and $280 \mathrm{ppm}$ (during interglacials) (Petit et al. 1999), an additional expansion of $\mathrm{C} 4$ vegetation could be observed every time $\mathrm{CO}_{2}$ levels dropped to $200 \mathrm{ppm}$ or below (Pinto et al. 2014). One can regard the emergence of $\mathrm{C} 4$ photosynthesis hence as a turning point in the history of our planet, in the sense that earth's biosphere will be entrenched, from now on till the end, in a battle over the ever declining amounts of recycled $\mathrm{CO}_{2}$ pumped out by earth's progressively receding geothermal activity (O'Malley-James et al. 2013). The total amount of
$\mathrm{CO}_{2}$ remaining in the atmosphere today, $0.05 \times 10^{18} \mathrm{~mol}$ at $280 \mathrm{ppm}$, is actually so small, that an individual $\mathrm{CO}_{2}$ molecule will remain in the air for only 5-10 years (Jacob 1999). Ever hungry plants are waiting (Zhu et al. 2016). The residence times for $\mathrm{O}_{2}$ and $\mathrm{N}_{2}$ molecules are, on the other side, $3 \mathrm{Ma}$ and 13 Ma respectively.

\subsection{Earth's lost billions}

Evolution is seldomly dependent on singular events. This holds also for major evolutionary steps like the invention of multicellularity and $\mathrm{C} 4$ photosynthesis, which have been developed independently, as discussed in Sect. 2.6 and 2.5, on at least 25 and 66 occasions respectively. Why did earth then take about one Ga to develop full fledged bacteria, and another one for the eukaryotic cell? It could not have been for the lack of living organisms.

It has been estimated in this context (Kallmeyer et al. 2012) that about $(9-32) \times 10^{29}$ bacteria dwell on earth, nowadays, making up in turn a few percent of the overall biomass. Given or taken a few orders of magnitude, we may assume that a similar number of microbes populated earth since the inception of life, with the population density being somewhat smaller before the invention of oxygenic photosynthesis in the late Archean (compare Sect. 2.4). $10^{29}$ organism with a life cycle of hours to days harbor an enormous evolutionary potential and a better understanding of the mechanism tapping into this evolutionary potential would hence help to clarify greatly our perspective of finding complex life elsewhere in the universe (Ward and Brownlec 2000). The alternative view taken here is that habitable planets would have a much higher chance to bear complex life if they would be given a speedup by several Ga.

\section{Habitability that waxes and wanes}

Transient habitability can be classified into four fundamental types (compare Fig. 4):

- fading habitability, when initially clement conditions become progressively adverse,

- delayed habitability, if the planet becomes habitable only late in the lifetime of the host star,

- punctuated habitability, if the habitability is interseeded by relatively short periods of inhabitable conditions and

- intermittent habitability, whenever clement conditions stabilize only intermittently.

Punctuated habitability is in this context the type of habitability taking a prominent place in doomsday scenarios. There is however a difference between catastrophic extinction and the temporarily termination of habitability per se. For a perspective we recall that the permian mass extinction at $252 \mathrm{Ma}$ (Benton and Benton 2003), the biggest extinction event ever occurring on earth, erased only 80-96\% 


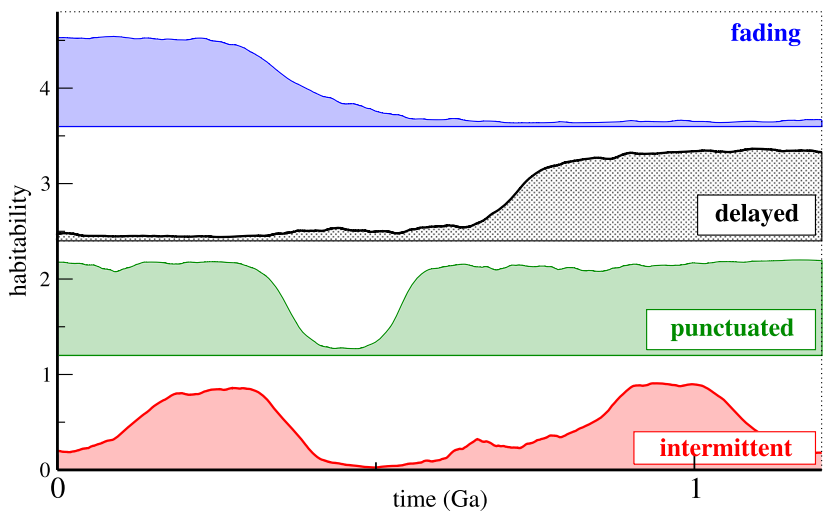

Fig. 4 Schematic illustration of the four possible classes of transient habitability. The timescale may range from (1-2) Ga for a FGK host star or up to $10 \mathrm{Ga}$ for a planet orbiting a $\mathrm{M}$ dwarf

of the marine and about $70 \%$ of the terrestrial vertebrate species. Biodiversity had no problems to recover in the aftermath within 8-9 Ma together with overall trophic levels (Chen and Benton 2012).

It is actually surprisingly hard to construct a scenario in which a singular event wipes multicellular life completely off the surface of a planet. The 1-1000 sec gamma-ray bursts emitted (possibly as frequent as once every $500 \mathrm{Ga}$ in our galactic habitat Piran and Jimenez 2014) during the collapse of a nearby massive star, have been discussed extensively in this context together with other conceivable high-energy astrophysical events (Horvath and Galante 2012). Such a burst would indeed sterilize half the planet, viz the exposed face, leading in addition to a series of events which would deplete the ozone layer for a few months by an average of $40 \%$ (Thomas et al. 2005). For comparison we note that the areas affected by the present-day antarctic ozone hole, on earth, which itself has had depletion levels of up to $50 \%$, have actually seen only a reduction of terrestrial plant productivity by less than $6 \%$ (Ballaré et al. 2011).

\subsection{Stagnant-lid planets}

Mantle and crustal reorganization processes are driven by the need to dissipate the internal heat. They often settle into steady-state convection patterns (Ernst 2009), with the two most important types being stagnant-lid and plate tectonics (compare Fig. 5).

Plate tectonics ensures on earth the continuous recycling of carbon, which would be otherwise lost as a consequence of the inevitable sedimentation of biomass and due to the ongoing carbonization of the oceanic lithosphere. Carbon is released back to the atmosphere from the subducted ocean floor in part directly and in part indirectly, through further mantel convection, by arc volcanos and by the mid-oceanic ridges respectively (Sleep et al. 2014). The oceanic lithosphere is such fully renewed within $170 \mathrm{Ma}$, recycling on

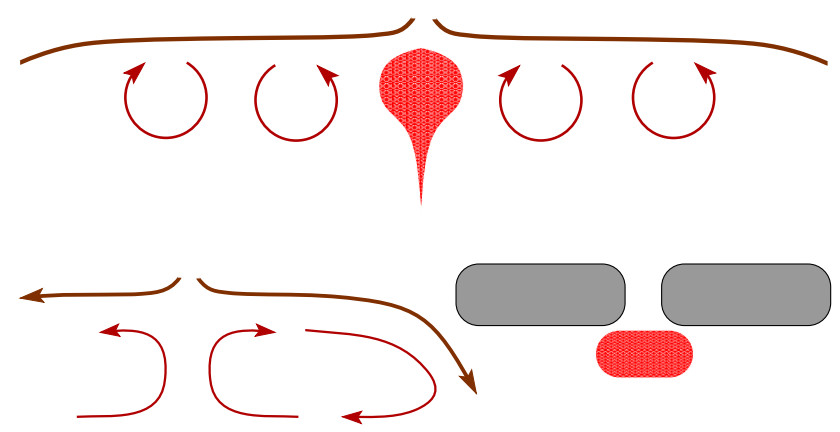

Fig. 5 Top: Illustration of stagnant-lid tectonics, for which lithosphere acts as a lid for the mantle dynamics. Updwelling plumes will however erupt through the lid time and again. Bottom: Illustration of plate tectonics. The ocean floor is subducted below the continental plates (grey), which tend in turn to act as heat traps. Mantle may overheat especially below a supercontinent, rise and erupt in the form of extended basaltic flooding

the way about $3.3 \times 10^{18} \mathrm{~mol}$ carbon per Ma (Sleep and Zahnle 2001). The sequestration of a 100 bar worth of carbon dioxide through the burial of carbonated crust, compare Sect. 2.1, was however achieved in the Hadean without the help of plate tectonics, which was then not yet operative (Harrison 2009).

Plate tectonics is the dominant but not the only type of tectonic activity present on our home planet. Other known processes are mid-plate volcanism, such as the one causing the Hawaiian and Yellowstone hotspots (Fouch 2012), and the subcontinental overheating of magma (or the updwelling of mantle plumes Santosh et al. 2014), which is thought to be causal for the widespread basaltic flooding occurring in conjunction with the breakup of supercontinents (Coltice et al. 2007).

Carbon recycling will be in contrast a much more dramatic process on planets with stagnant-lid tectonics, which do not dispose of a primary mechanism for the continuous recycling of $\mathrm{CO}_{2}$ (Lammer et al. 2009), but most probably of a discontinuous carbon cycle in the form of episodic basaltic overturns. The resulting fluctuations of atmospheric $\mathrm{CO}_{2}$ levels will however not forestall habitability per se (Petit et al. 1999), at least as long as no runaway instability is induced. It has been suggested in this context, that stagnantlid planets may have a more vigorous mantle dynamics than planets with plate tectonics (Kite et al. 2009) and that their volcanic activity may abate consequently somewhat faster. Stagnant-lid planets can therefore be expected to support clement conditions for extended but otherwise limited periods and to be hence prime candidates for the Genesis mission.

Stagnant-lid planets may of course also be utterly inhabitable whenever other factors won't allow it. The classical example is Venus, where the stagnant crust is punctuated continuously by updwelling plumes (Phillips and Hansen 1998), in part on a local and in part on a global scale (Sm- 
rekar et al. 2010). It is not known whether plate tectonics would have set in, eventually, in the case that Venus would not have lost its ocean.

\subsection{Hill instability}

A pair of planets is said to be Hill unstable when their orbits eventually cross due to their mutual gravitational interaction. The resulting massive orbital deformation (if not a direct collision) would terminate habitability for any planet located initially in a habitable zone. For an illustration of this process we consider a planetary system with two planets having masses $\mu_{i}(i=1,2$, relative to the mass of the host star) and eccentricities $e_{i}$ (a measure of how elliptic an orbit is). The Hill stability line is then determined by (Gladman 1993)

$$
\left(\mu_{1}+\frac{\mu_{2}}{\delta^{2}}\right)\left(\mu_{1} \gamma_{1}+\mu_{2} \gamma_{2} \delta\right)^{2}>\alpha^{3}+3^{4 / 3} \mu_{1} \mu_{2} \alpha^{5 / 3},
$$

where $\alpha=\mu_{1}+\mu_{2}$ is the total relative mass and $\gamma_{i}=$ $\sqrt{1-e_{i}^{2}}$. The distances of the two planets to the host stars are taken to be unity and $1+\Delta$ respectively, with $\delta=$ $\sqrt{1+\Delta}$.

Solving Eq. (4) for $e_{2}$ we have plotted in Fig. 6 the stability region for an earth- and Jupiter-like planetary system with $\mu_{2}=300 \mu_{1}$ and $\mu_{2}=1 / 1000$. The influence of $e_{1}$ is in this case so small that one can set $e_{1} \rightarrow 0$. Overlayed are the parameters of 129 known exoplanets around $\mathrm{G}$ and F stars whose orbits would not cross the orbit of a putative rocky planet located at $1 \mathrm{AU}$. Note, that the Hill instability line shown in Fig. 6 has been evaluated only for an outer planet of Jupiter mass. It moves up/down for outer planets with smaller/larger masses.

Most exoplanetary systems detectable to date are dominated by super-Jupiter gas giants. It is hence not surprising that configurations allowing for Hill stable habitable planets are rare. A certain time is however needed, the orbital lifetime, before the instability actually happens. This lifetime ranges from typically a few $10^{4}$ years, deep in the unstable region, to up to few $10^{9}$ years close to the Hill stability line (Rivera and Haghighipour 2007; Veras et al. 2013). Figure 6 hence suggests, that Genesis candidate planets may be found in systems with weak Hill instabilities.

Above considerations concerned exoplanets for which habitability is eventually terminated. The opposite may also happen, especially when two or more outer gas giants scatter dynamically (Veras and Armitage 2006). The resulting orbital deformations have been shown to move rocky planets closer to the sun (Veras and Armitage 2006), viz possibly from outside to inside the habitable zone (in the case that the initial distance was too large). A potentially large number of earth-size exoplanets may therefore be newcomers to their habitable zone, yet barren and hence candidates for a Genesis mission.

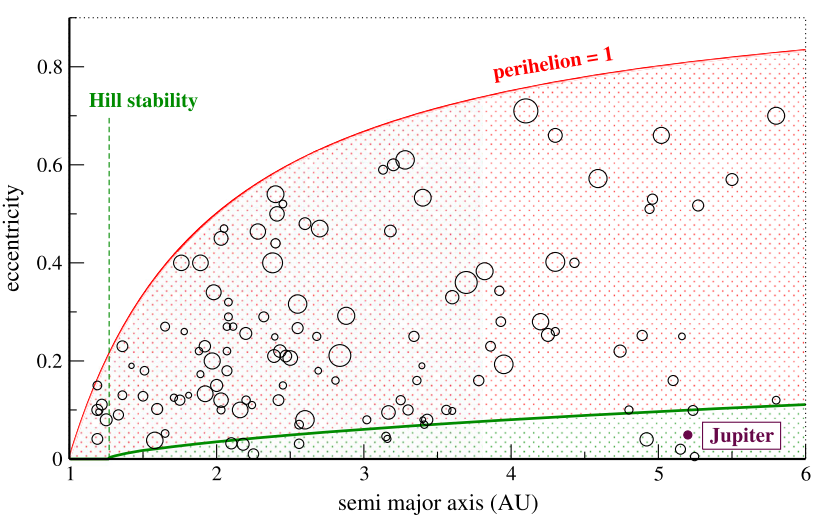

Fig. 6 The region of Hill stability (below the solid green line), as defined by Eq. (4, for an earth-like planet $\left(\mu_{2}=300 \mu_{1}\right)$ against the perturbation by a Jupiter-size planet $\left(\mu_{2}=10^{-3}\right)$ with eccentricity $e_{2}$. For large eccentricities the perihelion of the Jupiter-like planet would cross the orbit of the earth-like planet (solid red line). The open circles are 129 exoplanet around $\mathrm{G}$ and $\mathrm{F}$ stars listed in the extrasolar planets encyclopedia (Roques et al. 2016), with the sizes the circles indicating the respective masses. The filled maroon dot is the Jupiter of the solar system

\subsection{Lagrange instability}

Genesis candidate planets may also be found in Hill stable, but Lagrange unstable planetary systems. A massive orbital deformation occurs also in this case, for one of the involved planets, this time however one which leads either to a collision with the central star or to an escape from the planetary system. Estimating the Lagrange lifetimes for a specific exoplanetary system is a demanding task (Veras et al. 2016) and beyond the scope of the present study. We restrict ourselves therefore to a first assessment whether very long Lagrange lifetimes may potentially exist.

For this purpose we consider the minimal time $T_{L}$ for an Lagrange instability to occur. $T_{L}$ has been estimated (Veras and Mustill 2013) to scale roughly as

$\log _{10}\left(\frac{T_{L}}{T_{1}}\right) \sim 5.2\left(\frac{\mu}{\mu_{J}}\right)^{-0.18}$

for systems composed of two planets with an equal relative mass $\mu$ and eccentricities below $\sim 0.3 . T_{1}$ is here the orbital period of inner planet and $\mu_{J}$ the relative mass of the solar-system Jupiter. This scaling has been derived (Veras and Mustill 2013) for a configuration where the relative orbital distance of the two giants is confined to within $\left[1+0.3\left(1+e_{1}\right)\left(1+e_{2}\right)\right]$ Hill limits. The two gas giants are hence assumed to be in a Hill stable configuration close to the stability threshold.

In a Hill stable three-planet system, with an inner rocky planet in the habitable zone and two outer gas giants, a Lagrange instability of the inner gas giant occurring due to the mutual interaction between the two gas giants may also throw the rocky planet out of its orbit and consequently also 


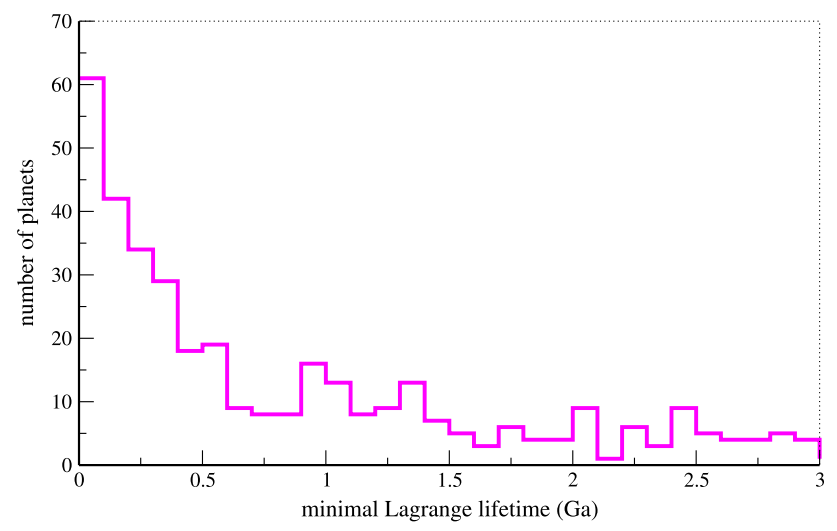

Fig. 7 The distribution of the minimal Lagrange lifetimes (in Ga; bin-size: $0.1 \mathrm{Ga}$ ), as given by Eq. (5), for 91 exoplanets around $\mathrm{G}$ and F stars, with eccentricities below 0.3 and with orbits outside $1 \mathrm{AU}$, listed in the extrasolar planets encyclopedia (Roques et al. 2016). The results are for putative exoplanetary systems, when a not too far away further-out Hill stable equal mass companion would exist in addition to each of the actually observed exoplanet

out of the habitable zone. We have hence evaluated Eq. (5) for all the 91 exoplanets shown in Fig. 6 having an eccentricity less than 0.3 . That is, we have added by hand to each of these exoplanets a putative equal mass companion located further out.

The distribution of Lagrange escape times resulting from this Gedanken-experiment is shown in Fig. 7. Small minimal Lagrange lifetimes clearly rule out the actual existence of a further-out (but close-by) equal mass companion in the respective exoplanetary system. Figure 7 shows on the other side that long Lagrange escape times would be present for suitable configurations of gas giants. Transient habitability as resulting from long-term Lagrange or other orbital instabilities, like resonances (see Sect. 2.2), of the host exoplanetary system may hence be common. A detailed analysis of the orbital past and future of extrasolar systems constitutes therefore an important screening tool for prospective Genesis missions.

\section{The Genesis mission}

A very large number of habitable planets may potentially exist in our galaxy. Current estimates range from about 0.06 per sun-like star (Petigura et al. 2013; Silburt et al. 2015), to $0.12-0.24$ habitable planets per M-dwarf (Dressing and Charbonneau 2015). The problem is however the distance. There are only 9 extrasolar systems (containing 14 stars) within 10 light years (lyr) and about $14 \times 10^{3}$ stars within 100 lyr (as extrapolated from the Gliese (Gliese and Jahreiß 1991) and HIPPARCOS (Perryman et al. 1997) catalogue entries). Among these we may expect up to a few hundred potentially habitable planets and possibly up to a few dozen Genesis candidate planets.

\subsection{Genesis vs. interstellar exploration}

Interstellar exploration via robotic or manned crafts can be regarded as a long-term research investment aiming to increase our scientific knowledge regarding the geophysics, the habitability and the astrobiology of extraterrestrial planetary systems (Crawford 2009). An explorative mission would therefore be only realizable if the projected mission duration would respect the maximal planning horizon of the funding institution, which will correlate in turn with typical human life expectancies, say a hundred years (Gilster 2015). The Genesis project is however not for human benefit and it is consequently irrelevant how long it would take for a Genesis craft to arrive to the target. A few millennia more or less would be negligible on evolutionary time scales.

- The absence of strict travel time requirements allows in first place for reduced cruising velocities. A Genesis probe would therefore need only comparatively modest financial and technical resources.

- The irrelevance of cruising times also allows to consider time-consuming deceleration options, e.g. via magnetic and/or electric drag (Zubrin and Andrews 1991; Perakis and Hein 2016).

- Overall traveling times are however restricted on the technical side by the lifetime of the constituent components.

A Genesis probe targeting candidate planets within a radius of 100 lyr may hence take a minimum of $10^{3}-10^{4}$ years to arrive. The Voyager spacecrafts need in comparison about $19 \times 10^{3}$ years to travel one light year. A close-up examination of the target planet, the first thing to be done, would then be followed by the decisive step, the autonomous decision whether to start the seeding sequence. The Genesis process would not be started if higher life forms would be detected from orbit.

The alternative to an in-situ decision taking, waiting for a response back from earth, would rely on the other hand on the long-term functioning of a trans-generational contract. Such a long-term conditioning would however be a questionable perspective when considering humanity's history of political and social turmoil. In this light it is actually an appealing aspect of the Genesis concept that the craft can be designed as a one-shot launch-and-forget project. The backtransmission of the in situ collected data (to anybody still listening) should be considered that notwithstanding to constitute an integral part of the mission layout.

\subsection{Biosphere incompatibilities}

Let us digress for a moment and ask the question: What may happen, if humanity's dream of a spaceship load of human settlers setting foot on a second earth would come true? In this case we would bring terrestrial life, microbes included, 
to a planet with a biosphere as rich as the one of our home planet. Both the alien biosphere and the invading fragment of the terrestrial biosphere would interpenetrate each other and humanity would have started a non-reversible experiment for which the outcome will most probably be determined by how universal the immune system of the respective multicellular organisms are.

The reason is that all multicellular organisms, plants and animals alike (Rodríguez et al. 2012), are vitally dependent on a performing immune system for their defense against pathogenic microbes. Key to the functioning of an immune reaction is the recognition of 'non-self', which is achieved in turn by the ability of the immune systems, at least on earth, to recognize certain products of microbial metabolism that are unique to microbiota (Medzhitov and Janeway 2002). How likely is it then, that 'non-self' recognition will work also for alien microbes?

Here we presume, that general evolutionary principles hold. Namely, that biological defense mechanisms evolve only when the threat is actually present and not just a theoretical possibility. Under this assumption the outlook for two clashing complex biospheres becomes quite dire.

- In the best case scenario the microbes of one of the biospheres will eat at first through the higher multicellular organism of the other biosphere. Primitive multicellular organism may however survive the onslaught through a strategy involving rapid reproduction and adaption. The overall extinction rates could then be kept, together with the respective recovery times, 1-10 $\mathrm{Ma}$ (Chen and Benton 2012), to levels comparable to that of terrestrial mass extinction events.

- In the worst case scenario more or less all multicellular organism of the planet targeted for human settlement would be eradicated. The host planet would then be reduced to a microbial slush in a pre-cambrian state, with considerably prolonged recovery times. The leftovers of the terrestrial and the indigenous biospheres may coexist in the end in terms of 'shadow biospheres' (Davies et al. 2009).

It is then clear, that exoplanets harboring multicellular life should be off-limit for Genesis missions. Expanding civilizations may find it equally unattractive to settle other life-bearing planets in the context of the Fermi paradox (Webb 2015; Gros 2005). Biosphere incompatibilities may be generic.

\subsection{Ethics and planetary protection}

Exoplanets already harboring a biosphere with multicellular lifeforms, being them primitive or advanced, would not be targeted by Genesis probes. The objective of the Genesis mission is after all to give life the chance to prosper in places where it has not yet a foothold, and not to invade and possibly to destroy existing biospheres (see Sect. 4.2). Regarding the ethics of such an endeavor one may ask whether it is legitimate to bring life to a planet which will cease anyhow to be habitable in the foreseeable future. Here we take the stance that death is no less part of the life cycle than birth, which is equivalent to saying that the value of being alive is not grounded in the avoidance of an inescapable death. We do acknowledge, however, that this is a viewpoint that will not be universally shared (somewhat related issues have been raised in the context of unlimited human life extensions Pecujlija 2013; Gyngell 2015).

The situation becomes substantially more tricky when primordial life forms do already exist on the candidate planet, in a stage either before or after the equivalent to the archean genetic expansion (which occurred on earth by 3.3-2.9 Ga, see Sect. 2.3). The Genesis process could then lead to the destruction of a substantial fraction of indigenous lifeforms and therefore to a flagrant violation of the current consensus regarding planetary protection (Nicholson et al. 2009). In contrast one may note that the microbes living on old earth, being them bacteria or eukaryotes, have never enjoyed human protection. Ethical or other type of arguments in favor of protecting our terrestrial microbes are generically not voiced. Taking a deeper look one may argue that planetary protection draws its justification from two sources (Lupisella 2009):

- The scientific benefit for humanity. Contaminating Mars or any other planet of the solar system with terrestrial microbes could ruin the possibility to study non-terrestrial lifeforms. This argument does not apply to Genesis candidate planets, which are selected expressively for being out or range for in depth science missions. Planetary protection will also break down, by the way, once the doors of a manned spaceship opens on Mars (Campion 2016).

- Independently evolved life constitutes a value per se (Randolph and McKay 2014). This argument is actually closely related to core motivation of the Genesis project, namely that life as such is valuable.

It then boils down to the balancing of two options involving the prospect that the habitable lifespan of the host planet may be too short for the indigenous life to evolve complex life by itself - the very reason the planet has been chosen whereas the further evolved precambrian terrestrial life may be prepared to do so.

\subsection{Seeding with unicellular organisms}

The simplest design for the seeding process would be the from-orbit delivery via nano-sized reentry capsules, which could be in turn ejected backwards at high velocities, viz decelerated with respect to the orbital motion of the main Genesis probe, by a compact railgun (Poniaev et al. 2015). A minimal heat shield would then be enough to protect the 
content of the delivery capsules during the subsequent drop to the surface.

Key to the success prospects of the Genesis mission is capability of the probe to use a databank of terrestrial genomes for the selection of the right mix of microbes to be synthesized in situ by the on-board gene laboratory. This brew of prokaryotes (bacteria) and unicellular eukaryotes will be optimized with respect to the requirements resulting from the geophysical conditions of the host planet. It could be advantageous for the Genesis probe to spread the seeding process over several centuries, albeit with slowly adapting mixtures of microbes.

The goal of the Genesis mission is to fast forward the target planet to a precambrian state (see Fig. 1). Life would be given a head start consisting of a biosphere of unicellular organisms, from which on it could further flourish and develop. Direct seeding with multicellular organisms wouldn't be impossible per se, but both substantially more complex and in part also questionable.

- A planet with substantial levels of $\mathrm{O}_{2}$ may be expected to develop complex life on its own. Planetary protection arguments in conjunction with possible biosphere incompatibilities would then dictate not to bring higher life forms to its surface (nor to seed it in first place).

- A planet without $\mathrm{O}_{2}$, the most probable situation, could also be seeded with multicellular life, as a matter of principle, but only with fully anaerobic animals of the type that are thought to dwell in earth's oxygen-free deep sea environments (their cells are devoid of mitochondria, possessing however hydrogenosomes Mentel and Martin 2010). It is however questionable whether a passively dropping reentry probe could successfully deliver these sub-millimeter animals to their proper habitats.

Even though most eukaryotes are aerobic and hence dependent on free oxygen, a wide range of unicellular eukaryotes have adapted to anoxic environments (Müller et al. 2012). Seeding of a planet devoid of free oxygen with eukaryotes will hence not pose a problem (Yu et al. 2012).

\subsection{Post-seeding evolution and the oxidation of the atmosphere}

The mission of the Genesis project is to lay the foundations for a self-evolving biosphere. It is however clear that fine tuning won't be possible and that the primary seeding process will result at best in a highly unbalanced ecosystems of microbes. Global scale 'ecological disasters' are hence expected to occur initially in the post-seeding phase, such as the uncontrolled blossoming of unicellular algae. The ecosystem should however self-stabilize relatively fast, say within a few thousand years. The further evolution will then depend on a flurry of parameters, like the initial concentration of atmospheric $\mathrm{CO}_{2}$, the average temperature, the eventual presence of continents and the overall level of hydrothermal activity.

It is presently not possible to estimate reliably how long it will take afterwards for the photosynthetically produced $\mathrm{O}_{2}$ to accumulate in the atmosphere of a Genesis plant.

- Nearly all excess oxygen ever produced by earth's biosphere has been used oxidizing the crust, compare Fig. 3, with less than 1/34 accumulating in the end in the atmosphere.

- Planets may dispose of quite different crustal compositions in terms of the relative percentages of oxygen and reducing elements (see Table 1).

- Both the bare metallicity (the abundance of heavy elements) and the relative abundances of the heavy elements will be distinct to a planetary system (Longstaff 2014).

- The initial core-crust segregation will depend likewise on the then present conditions, like the overall mass of the planet and the amount of radioactive heating (Longstaff 2014).

- The post-segregation deposition of elements by comets and asteroids, see Sect. 2.2, may also influence the composition of the crust.

- Non-biological processes like

$$
2 \mathrm{FeO}+\mathrm{H}_{2} \mathrm{O} \rightarrow \mathrm{Fe}_{2} \mathrm{O}_{3}+\mathrm{H}_{2}
$$

contribute additionally to the net oxidation of the crust whenever the resulting $\mathrm{H}_{2}$ molecule manages to escape to space (Wallace and Hobbs 2006).

About $60 \%$ of all atoms present in the crust of the earth are oxygen atoms and one may wonder whether this percentage is already close to saturation, at least with respect of what may be achievable by geo-planetary processes. It would be in any case optimal if the oxidation of the crust through antecedenting inorganic processes would be in an advanced stage. This could be expected to be the case for planets with delayed habitability and with elevated stratospheric $\mathrm{H}_{2} \mathrm{O}$ concentrations (Catling et al. 2001), allowing in turn for hydrogen to escape into space (see the analogous discussion in Sect. 2.1).

A constant and hopefully high flux of minerals and $\mathrm{CO}_{2}$ is a general precondition for a Genesis planet to develop a high bioproductivity and hence a prerequisite also for a potentially rapid raise of atmospheric oxygen levels. We note here that about $11 \times 10^{18}$ out of the $8700 \times 10^{18}$ mol of organically produced $\mathrm{C}$ per Ma are buried in continental sediments (Field et al. 1998; Sleep and Zahnle 2001), nowadays on earth, from where it is recycled through carbonate weathering within about $350 \mathrm{Ma}$ (compare also Fig. 3). With about $37 \times 10^{18} \mathrm{~mol} \mathrm{O}_{2}$ present in the atmosphere (Jacob 1999) that would imply that an atmosphere worth of $\mathrm{O}_{2}$ is produced via organic $\mathrm{CO}_{2}$ fixation every $37 / 11=3.4 \mathrm{Ma}$. 
The balance of the biotic oxygen production through the weathering of continental carbon deposits occurring on earth will however not start immediately on planets not yet disposing of extended carbon sediments. The pace at which the atmospheric $\mathrm{O}_{2}$ level rises then depends on the overall bioproductivity and on the amount of oxygen lost to the crust. An appreciable level could be achieved relatively fast, within 10-100 Ma, if comparatively small amounts of oxygen would be lost, viz whenever the crust of the Genesis planet would already be in an advanced state of oxidation. The initial surge of oxygen levels would be rebalanced in this scenario only once the weathering rates have caught up respectively.

Most habitable planets will probably take of the order of a few Ga to acquire an oxygen bearing atmosphere, if at all. We are however confident that substantially shorter time scales may be achievable, as discussed above, under optimal conditions and that we will hence be able to find Genesis candidate planets for which an initial seeding would initiate a geo-evolutionary process leading to the subsequent emergence of complex and multicellular life. Our best case estimates however still exceed human planning horizons by many orders of magnitude, implying that the Genesis process is intrinsically unsuited for the preparation of a barren planet for an eventual human colonization.

\section{Conclusions}

Today's scientific environment is made up by a diverse mix of emerging and mature fields, characterized respectively by swift and lackluster rates of progress (Gros 2012). The sluggish progress of traditional space launching technologies (Ragab et al. 2015) contrast here, e.g., with the rapid advances in synthetic biology (Stano and Luisi 2013; Caspi and Dekker 2014). Transformative concepts are hence critical for reigniting innovation in science and technology time and again. It has been proposed in this context(Benford 2013), that robotic interstellar missions of low-weight crafts accelerated by beams of directed energy will become realizable, both on technical grounds and financially, in the near future (Gilster 2015; Worden et al. 2016). At the same time we are discovering that planetary habitability isn't an all-or-nothing feature characterizing exoplanets (Güdel et al. 2014). Our galaxy is expected in particular to teem with planets which are in part habitable, but for which the clement conditions do not last long enough for higher life forms to evolve on their own.

Reversing the argument we have pointed out in this study that complex life may emerge also on transiently habitable exoplanets whenever the extraordinary long time it took earth to develop eukaryotic cells could be leapfrogged. We have argued furthermore that this endeavor could be achieved by a light-weight interstellar craft using a robotic gene laboratory for the seeding the target exoplanet with a brew of in situ synthesized microbes. By the end of the mission, which we call the Genesis project, a precambrian and hopefully thriving biosphere of unicellular organisms would flourish on the candidate planet. Complex life in the form of multicellular animals and plants will evolve autonomously at a later state once the photosynthetically produced oxygen has had the time to accumulate in the atmosphere.

One of the key issues remaining to be settled at this stage regards the selection procedure for target planets. Remote sensing of exo-planetary biosignatures from earth is possible (Des Marais et al. 2002), albeit only to a certain degree. An even more daring task would be to actually prove that a world is uninhabited (Persson 2014). It is hence clear that the final decision to go ahead must be taken autonomously by the on-board artificial intelligence. This may seem an imprudent strategy nowadays, but possibly not so in a few decades.

The Genesis mission is furthermore unique in the sense that the actual cruising velocity is of minor importance. It could be launched with the help of suitable beams of directed energy and decelerated at arrival by time consuming passive means like magnetic sails. We hence believe that the Genesis project opens a new venue for interstellar missions and for the unfolding of life in our galactic surroundings.

Open Access This article is distributed under the terms of the Creative Commons Attribution 4.0 International License (http://creative commons.org/licenses/by/4.0/), which permits unrestricted use, distribution, and reproduction in any medium, provided you give appropriate credit to the original author(s) and the source, provide a link to the Creative Commons license, and indicate if changes were made.

\section{References}

Bada, J.L.: Earth Planet. Sci. Lett. 226, 1 (2004)

Ballaré, C., Caldwell, M., Flint, S., Robinson, S.A., Bornman, J.: Photochem. Photobiol. Sci. 10, 226 (2011)

Benford, J.: J. Br. Interplanet. Soc. 66, 85 (2013)

Benton, M.J., Benton, M.: When Life Nearly Died: The Greatest Mass Extinction of All Time, vol. 5. Thames \& Hudson London, New York (2003)

Blair, J.E., Hedges, S.B.: Mol. Biol. Evol. 22(3), 387 (2005)

Bottke, W.F., et al.: Nature 485, 78 (2012)

Brashears, T., et al.: In: SPIE Optical Engineering + Applications, vol. 9616,2015

Campion, N.: Commercial Space Exploration: Ethics, Policy and Governance, 9 (2016)

Canfield, D.E.: Annu. Rev. Earth Planet. Sci. 33, 1 (2005)

Canup, R.M., Righter, K.: Origin of the Earth and Moon, vol. 30. University of Arizona Press, Tucson (2000)

Caspi, Y., Dekker, C.: Syst. Synth. Biol. 8, 249 (2014)

Catling, D.C., Claire, M.W.: Earth Planet. Sci. Lett. 237, 1 (2005)

Catling, D.C., Zahnle, K.J., McKay, C.P.: Science 293, 839 (2001)

Chen, Z.-Q., Benton, M.J.: Nat. Geosci. 5, 375 (2012)

Collatz, G.: Planta 134, 127 (1977) 
Coltice, N., Phillips, B., Bertrand, H., Ricard, Y., Rey, P.: Geology 35, 391 (2007)

Crawford, I.: J. Br. Interplanet. Soc. 62, 415 (2009)

David, L.A., Alm, E.J.: Nature 469, 93 (2011)

Davies, P.C., Benner, S.A., Cleland, C.E., Lineweaver, C.H., McKay, C.P., Wolfe-Simon, F.: Astrobiology 9(2), 241 (2009)

Des Marais, D.J.: Science 289, 1703 (2000)

Des Marais, D.J., Harwit, M.O., Jucks, K.W., Kasting, J.F., Lin, D.N., Lunine, J.I., Schneider, J., Seager, S., Traub, W.A., Woolf, N.J.: Astrobiology 2(2), 153 (2002)

Drake, M.J., Righter, K.: Nature 416, 39 (2002)

Dressing, C.D., Charbonneau, D.: Astrophys. J. 807, 45 (2015)

Ernst, W.: Gondwana Res. 15, 243 (2009)

Field, C.B., Behrenfeld, M.J., Randerson, J.T., Falkowski, P.: Science 281, 237 (1998)

Fouch, M.J.: Geology 40, 479 (2012)

Gibson, D.G., et al.: Science 329, 52 (2010)

Gilster, P.: The Dragonfly Project (2015). http://www.centauri-dreams. org/?p=33615

Gladman, B.: Icarus 106, 247 (1993)

Gliese, W., Jahreiß, H.: Brotzmann, L.E., Gesser, S.E. (eds.) On the Astronomical Data Center CD-ROM: Selected Astronomical Catalogs, vol. I, NASA/Astronomical Data Center, Goddard Space Flight Center, Greenbelt, MD 1 (1991)

Gomes, R., Levison, H.F., Tsiganis, K., Morbidelli, A.: Nature 435, 466 (2005)

Goodenough, U., Heitman, J.: Cold Spring Harb. Perspect. Biol. 6, 016154 (2014)

Gros, C.: J. Br. Interplanet. Soc. 58, 108 (2005)

Gros, C.: Complex Syst. 21, 183 (2012)

Grosberg, R.K., Strathmann, R.R.: Annu. Rev. Ecol. Evol. Syst. 38, 621 (2007)

Güdel, M., et al.: Protostars and Planets VI, vol. 1, p. 883 (2014)

Gyngell, C.: J. Med. Philos. 40, 696 (2015)

Hamano, K., Abe, Y., Genda, H.: Nature 497, 607 (2013)

Harrison, T.M.: Annu. Rev. Earth Planet. Sci. 37, 479 (2009)

Horvath, J.E., Galante, D.: Int. J. Astrobiol. 11, 279 (2012)

Hutchison, C.A., et al.: Science 351, 6253 (2016)

Igamberdiev, A.U., Lea, P.J.: Photosynth. Res. 87, 177 (2006)

Jacob, D.: Introduction to Atmospheric Chemistry. Princeton University Press, Princeton (1999)

Jones, B.W., Sleep, P.N., Underwood, D.R.: Astrophys. J. 649, 1010 (2006)

Kallmeyer, J., Pockalny, R., Adhikari, R.R., Smith, D.C., D'Hondt, S.: Proc. Natl. Acad. Sci. 109, 16213 (2012)

Kasting, J.F., Chen, H., Kopparapu, R.K.: Astrophys. J. Lett. 813, 3 (2015)

Kaufman, A.J., Xiao, S.: Nature 425, 279 (2003)

Kite, E.S., Manga, M., Gaidos, E.: Astrophys. J. 700, 1732 (2009)

Knoll, A.H., Javaux, E.J., Hewitt, D., Cohen, P.: Philos. Trans. R. Soc. Lond. B, Biol. Sci. 361, 1023 (2006)

Korenaga, J.: Annu. Rev. Earth Planet. Sci. 41, 117 (2013)

Lammer, H., et al.: Astron. Astrophys. Rev. 17, 181 (2009)

Laskar, J., Joutel, F., Robutel, P.: Nature 361, 615 (1993)

Leconte, J., Forget, F., Charnay, B., Wordsworth, R., Pottier, A.: Nature 504, 268 (2013)

Lide, D.R., Fredrikse, H.: CRC Handbook of Chemistry and Physics. CRC Press, Boca Raton (2008)

Lodders, K., Palme, H., Gail, H.-P.: In: Solar system. LandoltBörnstein: Numerical Data and Functional Relationships in Science and Technology, vol. 4B, p. 712. Springer, Berlin (2009)

Long, K.F.: Deep Space Propulsion: A Roadmap to Interstellar Flight. Springer, New York (2011)

Longstaff, A.: Astrobiology: An Introduction. CRC Press, Boca Raton (2014)
Lupisella, M.: Exploring the Origin, Extent, and Future of Life: Philosophical, Ethical and Theological Perspectives, 4, 186 (2009)

Lyons, T.W., Reinhard, C.T., Planavsky, N.J.: Nature 506, 307 (2014)

Macdonald, F.A., et al.: Science 327, 1241 (2010)

Marshall, C.R.: Annu. Rev. Earth Planet. Sci. 34, 355 (2006)

McDonough, W.F., Sun, S.-S.: Chem. Geol. 120, 223 (1995)

Medzhitov, R., Janeway, C.A.: Science 296, 298 (2002)

Mentel, M., Martin, W.: BMC Biol. 8, 1 (2010)

Morris, S.C.: Proc. Natl. Acad. Sci. 97, 4426 (2000)

Müller, M., et al.: Microbiol. Mol. Biol. Rev. 76, 444 (2012)

Nicholson, W.L., Schuerger, A.C., Race, M.S.: Trends Microbiol. 17, 389 (2009)

Nisbet, E., Sleep, N.: Nature 409, 1083 (2001)

Och, L.M., Shields-Zhou, G.A.: Earth-Sci. Rev. 110, 26 (2012)

O’Malley-James, J.T., Greaves, J.S., Raven, J.A., Cockell, C.S.: Int. J. Astrobiol. 12, 99 (2013)

Parfrey, L.W., Lahr, D.J., Knoll, A.H., Katz, L.A.: Proc. Natl. Acad. Sci. 108, 13624 (2011)

Pecujlija, M.: Eur. J. Sci. Theol. 9, 161 (2013)

Perakis, N., Hein, A.M.: Preprint (2016). arXiv:1603.03015

Perryman, M.A., Lindegren, L., Kovalevsky, J., Hoeg, E., Bastian, U., Bernacca, P., Crézé, M., Donati, F., Grenon, M., Grewing, M., et al.: Astron. Astrophys. 323, 49 (1997)

Persson, E.: Challenges 5(2), 224 (2014)

Petigura, E.A., Howard, A.W., Marcy, G.W.: Proc. Natl. Acad. Sci. 110, 19273 (2013)

Petit, J.-R., et al.: Nature 399, 429 (1999)

Phillips, R.J., Hansen, V.L.: Science 279, 1492 (1998)

Pinto, H., Sharwood, R.E., Tissue, D.T., Ghannoum, O.: J. Exp. Bot. 65, 3669 (2014)

Piran, T., Jimenez, R.: Phys. Rev. Lett. 113, 231102 (2014)

Poniaev, S., et al.: Acta Astronaut. 109, 162 (2015)

Ragab, M.M., Cheatwood, F.M., Hughes, S.J., Lowry, A.: In: AIAA SPACE 2015 Conference and Exposition, p. 4490 (2015)

Randolph, R.O., McKay, C.P.: Int. J. Astrobiol. 13, 28 (2014)

Rasmussen, B., Fletcher, I.R., Brocks, J.J., Kilburn, M.R.: Nature 455, 1101 (2008)

Rivera, E., Haghighipour, N.: Mon. Not. R. Astron. Soc. 374, 599 (2007)

Roberts, N.M.: Geosci. Front. 4, 681 (2013)

Rodríguez, R.M., López-Vázquez, A., López-Larrea, C.: In: Sensing in Nature, p. 237. Springer, New York (2012)

Roques, F., et al.: The Extrasolar Planets Encyclopaedia (2016). http:// exoplanet.eu/catalog

Rota-Stabelli, O., Daley, A.C., Pisani, D.: Curr. Biol. 23, 392 (2013)

Rushby, A.J., Claire, M.W., Osborn, H., Watson, A.J.: Astrobiology 13, 833 (2013)

Sage, R.F., Sage, T.L., Kocacinar, F.: Annu. Rev. Plant Biol. 63, 19 (2012)

Santosh, M., Maruyama, S., Sawaki, Y., Meert, J.G.: Gondwana Res. 25, 945 (2014)

Shapiro, J.A., Dworkin, M., et al.: Bacteria as Multicellular Organisms. Oxford University Press, Oxford (1997)

Sheldon, N.D.: Precambrian Res. 147, 148 (2006)

Silburt, A., Gaidos, E., Wu, Y.: Astrophys. J. 799, 180 (2015)

Sleep, N.H.: Cold Spring Harb. Perspect. Biol. 2, 002527 (2010)

Sleep, N.H., Zahnle, K.: J. Geophys. Res., Planets 106, 1373 (2001)

Sleep, N.H., Zahnle, K.J., Lupu, R.E.: Philos. Trans. R. Soc., Math. Phys. Eng. Sci. 372, 20130172 (2014)

Smrekar, S.E., et al.: Science 328, 605 (2010)

Speijer, D., Lukeš, J., Eliáš, M.: Proc. Natl. Acad. Sci. 112, 8827 (2015)

Stano, P., Luisi, P.L.: Curr. Opin. Biotechnol. 24, 633 (2013)

Tang, H., Chen, Y.: Geosci. Front. 4, 583 (2013)

Thomas, B.C., et al.: Astrophys. J. 634, 509 (2005)

Tsiganis, K., Gomes, R., Morbidelli, A., Levison, H.: Nature 435, 459 (2005) 
Veras, D., Armitage, P.J.: Astrophys. J. 645, 1509 (2006)

Veras, D., Mustill, A.J.: Mon. Not. R. Astron. Soc. Lett. 434, 11 (2013)

Veras, D., Mustill, A.J., Bonsor, A., Wyatt, M.C.: Mon. Not. R. Astron. Soc. 431, 1686 (2013)

Veras, D., et al.: Mon. Not. R. Astron. Soc. 476 (2016). doi:10.1093/ mnras/stw476

Wächtershäuser, G.: Philos. Trans. R. Soc. Lond. B, Biol. Sci. 361, 1787 (2006)

Wallace, J.M., Hobbs, P.V.: Atmospheric Science: an Introductory Survey, vol. 92. Academic press, San Diego (2006)

Ward, P.D., Brownlec, D.: Rare Earth: Why Complex Life Is Uncommon in the Universe. Springer, New York (2000)
Webb, S.: If the Universe Is Teeming with Aliens... Where Is Everybody? Seventy-Five Solutions to the Fermi Paradox and the Problem of Extraterrestrial Life. Springer, Heidelberg (2015)

Worden, P., et al.: The starshot project of breakthrough initiatives (2016). http://breakthroughinitiatives.org/Initiative/3

Yu, K.H., et al.: J. Endocytobiosis Cell Res. 64, 68 (2012)

Zhang, X., et al.: Gondwana Res. 25, 896 (2014)

Zhang, Q., Lubin, P.M., Hughes, G.B., Melis, C., Walsh, K.J.: In: SPIE Optical Engineering + Applications, vol. 9616, 2015

Zhu, Z., et al.: Nat. Clim. Change (2016). doi:10.1038/nclimate3004

Zubrin, R.M., Andrews, D.G.: J. Spacecr. Rockets 28, 197 (1991) 